\section{BRAZIULIAN JOURNAL}

OF MEDICAL AND BIOLOGICAL RESFARCH

www.bjournal.com.br
ISSN 0100-879X

Volume 43 (9) 812-913 September 2010

BIOMEDICAL SCIENCES

AND

CLINICAL INVESTIGATION

Braz J Med Biol Res, September 2010, Volume 43(9) 906-909

doi: 10.1590/S0100-879X2010007500086

Participation of endogenous opioids in the antinociception induced by resistance exercise in rats

G.S. Galdino, I.D.G. Duarte and A.C. Perez

The Brazilian Journal of Medical and Biological Research is partially financed by
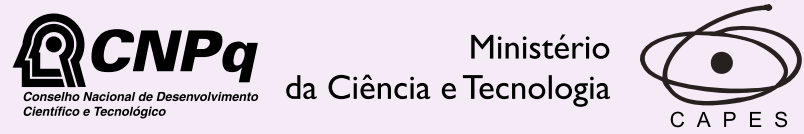

Ministério da Educação

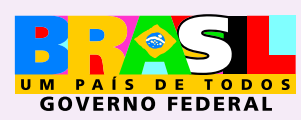

DFAPESP

Institutional Sponsors
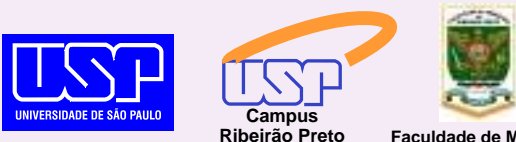

$\oplus$ SHIMADZU

GE Healthcare
Hotsite of proteomics metabolomics developped by:

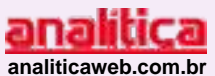

Thermo
SCIEN TIFIC 


\title{
Participation of endogenous opioids in the antinociception induced by resistance exercise in rats
}

\author{
G.S. Galdino, I.D.G. Duarte and A.C. Perez \\ Departamento de Farmacologia, Instituto de Ciências Biológicas, \\ Universidade Federal de Minas Gerais, Belo Horizonte, MG, Brasil
}

\begin{abstract}
Exercise is a low-cost intervention that promotes health and contributes to the maintenance of the quality of life. The present study was designed to investigate the influence of different resistance exercise protocols on the nociceptive threshold of rats. Female Wistar rats were used to perform exercises in a weight-lifting exercise model. The following groups were examined $(\mathrm{N}$ = 6 per group): untrained rats (control group); an acute protocol group consisting of rats submitted to 15 sets of 15 repetitions of resistance exercise (acute group); rats exercised with 3 sets of 10 repetitions, three times per week for 12 weeks (trained group), and a group consisting of trained rats that were further submitted to the acute protocol (trained-acute group). The nociceptive threshold was measured by the paw-withdrawal test, in which the withdrawal threshold (escape reaction) was measured by an apparatus applying force to the plantar surface of the animal paw. The opioid antagonist naloxone $(2 \mathrm{mg} / \mathrm{kg}) \mathrm{was}$ administered subcutaneously $10 \mathrm{~min}$ before the exercise protocols. The trained group demonstrated antinociception only up to day 45 of the 12 -week training period. A significant increase $(37 \%, \mathrm{P}<0.05)$ in the nociceptive threshold was produced immediately after exercise, decreasing to $15 \%$ after $15 \mathrm{~min}$, when the acute exercise protocol was used. Naloxone reversed this effect. These data show that the acute resistance exercise protocol was effective in producing antinociception for 15 min. This antinociceptive effect is mediated by the activation of opioid receptors.
\end{abstract}

Key words: Resistance exercise; Opioid receptors; Antinociception

\section{Introduction}

Exercise may serve as an effective and healthy pain management alternative and resistance exercise may be very helpful for treating patients with impaired exercise capacity due to both peripheral maladaptations of the skeletal musculature and/or cardiac limitations. Programmed and segmented periods of training (periodized resistance training) for 16 weeks increased strength, reduced pain and disability, and improved the quality of life of middle-aged and elderly males (1). Other studies have reported the amelioration of pain in patients with intermittent claudication and subacromial impingement syndrome after strength training $(2,3)$. The rat hind limb muscle strength training experiment is an animal model analogous to human weightlifting training. It is a specialized method of conditioning, designed to increase muscle strength, muscle endurance and muscle power.

Numerous studies have demonstrated a direct relation- ship between a reduction in cutaneous and dental pain and an increase in $\beta$-endorphins in plasma after aerobic exercise $(4,5)$; however, few have investigated analgesia induced by resistance exercise. Furthermore, none of these were performed with rodents or evaluated the mechanisms responsible for this effect. Therefore, the aim of the present study was to investigate the effect of different resistance exercise protocols on the nociceptive threshold in rats and the participation of opioid receptors.

\section{Material and Methods}

\begin{abstract}
Animals
Female Wistar rats weighing 230-250 g obtained from the Federal University of Minas Gerais (UFMG), Brazil, were housed in individual cages and kept at a constant temperature of $23^{\circ} \mathrm{C}$ with a 12-h light-dark cycle and free
\end{abstract}


access to food and water.

The study was conducted in concordance with the IASP Guidelines on use of laboratory animals and all experiments were approved by the Ethics Committee for Animal Experimentation of the Federal University of Minas Gerais (Protocol \#185/2007).

\section{Exercise}

Resistance exercise was performed using a weightlifting exercise model (6). Rats fitted with a canvas jacket were able to regulate the twisting and flexing of their torsos and were suspended in a standard position on their hind limbs. The rats flexed their legs repeatedly, which lifted the weight-arm of the training apparatus. In each group, the exercise started after 2 weeks of adaptation and after measurement of the maximum weight lifted (1 repetition maximum) with the squat-training apparatus, and the training load was set at 65 to $75 \%$ of 1 repetition maximum (RM). In the trained protocol, every 2 weeks $1 \mathrm{RM}$ was redetermined and the training load raised accordingly.

The rats were randomly divided into the following groups ( $N=6$ per group): control (Co): rats that were not exercised and received saline; trained ( $\mathrm{Tr}$ ): rats that were exercised with 3 sets of 10 repetitions, each with a 120-s rest period between sets, three times per week for 12 weeks and received saline; acute $(\mathrm{Ac})$ : rats that were exercised with 15 sets of 15 repetitions each with a 120-s rest between sets and received saline, and another group that received naloxone $(\mathrm{Ac}+\mathrm{Nx})$. Trained-acute $(\mathrm{Tr}-\mathrm{Ac})$ : Tr rats that were submitted to the Ac protocol (7).

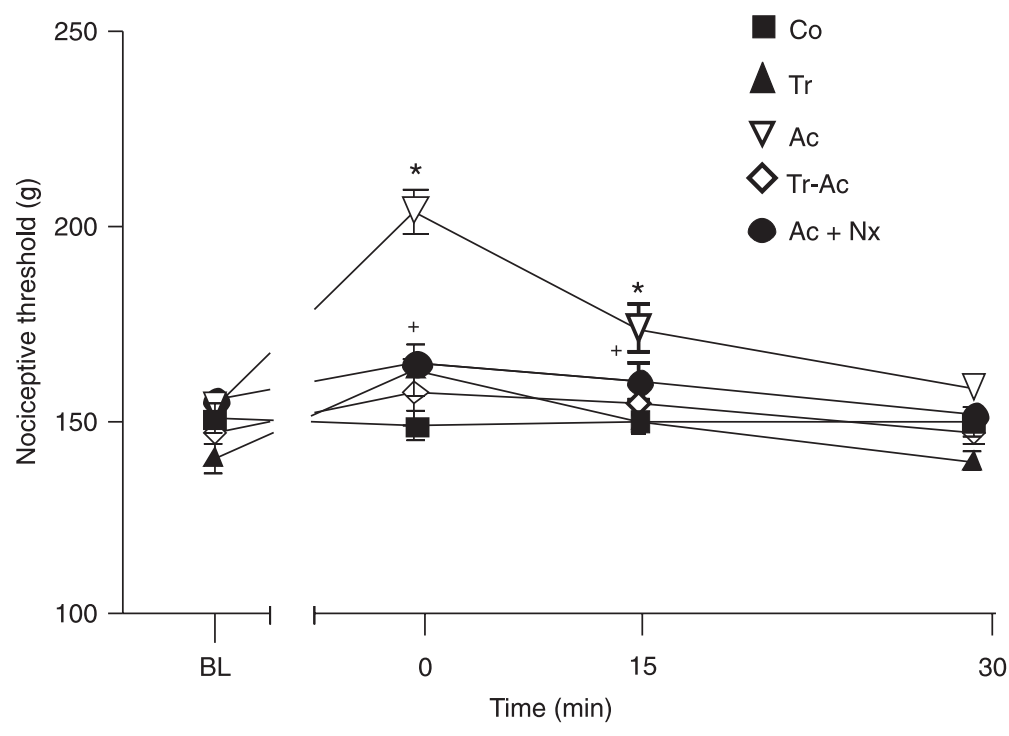

Figure 1. Effect of different resistance exercise protocols on the nociceptive threshold of the following groups of rats ( $\mathrm{N}=6$ per group): control (Co), trained $(\mathrm{Tr})$, acute $(\mathrm{Ac})$, trained-acute $(\mathrm{Tr}-\mathrm{Ac})$, and naloxone $(2 \mathrm{mg} / \mathrm{kg})$ injected, subcutaneously, before acute exercise ( $\mathrm{Ac}+\mathrm{Nx}$; $\mathrm{BL}=$ baseline latency. ${ }^{*} \mathrm{P}<0.05 \mathrm{Ac}$ vs $\mathrm{Co}$ and ${ }^{+} \mathrm{P}<0.05 \mathrm{Ac}+\mathrm{Nx}$ vs Ac (one-way ANOVA followed by Bonferroni's test).

\section{Nociceptive test}

Nociceptive threshold was evaluated by the paw-withdrawal test: an apparatus (Ugo Basile, Italy) was used to measure the withdrawal threshold. A cone-shaped presser with a rounded tip was applied to the plantar surface of the paw. The frequency of force application was set at 150-160 $\mathrm{g} / \mathrm{s}$ and there was a $240 \mathrm{~g} / \mathrm{s}$ cut-off loading to avoid damaging the tissue. The intensity of pressure causing an escape reaction was defined as the withdrawal threshold (8).

Nociception was measured before and immediately after exercise and every $15 \mathrm{~min}$, while the analgesic effect produced by exercise was present. In the trained group, the nociceptive threshold was measured every 15 days during the 12-week training period.

\section{Drugs}

Naloxone (2 mg/kg; Sigma, USA), dissolved in 0.9\% sterile saline, was administered subcutaneously $10 \mathrm{~min}$ before acute exercise $(\mathrm{Ac}+\mathrm{Nx}$ ) and also to a group that did not perform exercise (data not shown).

\section{Statistical analysis}

Data are reported as means \pm SEM and were analyzed by one-way ANOVA followed by Bonferroni's test, with the level of significance set at $P<0.05$.

\section{Results}

The acute protocol (Ac) produced a significant increase $(P<0.05)$ in the nociceptive threshold for 15 min compared to the control group ( $\mathrm{Co}$; Figure 1). The other resistance exercise protocols did not modify the nociceptive threshold of the rats compared to Co. The antinociception produced by Ac was significantly reversed $(P<0.05)$ by the opioid antagonist naloxone $(\mathrm{Ac}+\mathrm{Nx})$. Naloxone alone did not produce any alteration in the nociceptive threshold (data not shown). This group was included in the experimental design in order to exclude any intrinsic effect of naloxone on the nociceptive response.

Figure 2 demonstrates that exercise was effective in increasing the nociceptive threshold measured immediately after exercise, up to day 45 of the 12-week training period.

\section{Discussion}

The resistance exercise acute protocol (Ac) significantly increased nociceptive threshold for $15 \mathrm{~min}$. The present study is the first to evaluate antinociception induced by resistance exercise in rats, mediated by the activation of opioid receptors. Two 
studies conducted on humans have reported analgesia after resistance exercise, but the subjects had previous resistance exercise experience $(9,10)$. In the present study, however, the resistance exercise protocols involving trained rats ( $\mathrm{Tr}, \mathrm{Tr}-\mathrm{Ac})$ did not produce antinociception. A similar result with trained humans was obtained in one study, where the participants were submitted to resistance exercise for 12 weeks after which they presented minimal pain tolerance (11).

Several studies have shown that endogenous opioids are mainly responsible for exerciseinduced analgesia $(5,12)$. Our findings are consistent with these studies because we used the opioid antagonist naloxone to revert the antinociception produced by the acute exercise protocol (Ac).

Endogenous opioids may act by modulating pain at peripheral and central levels (13). Many studies have demonstrated an increase in the plasma levels of endogenous opioids, mostly $\beta$-endorphin, during and after aerobic exercise (5); however, only a few studies have investigated alterations in this parameter when resistance exercise was involved, with equivocal results. Analysis of blood samples of athletes submitted to 3 sets of 8 repetitions at $80 \%$ of 1 RM showed no significant change in the circulating $\beta$-endorphin levels (14), while a significant increase in plasma $\beta$-endorphin levels was detected in men after a high total work-exercise protocol (15).

Training ( $\mathrm{Tr}$ ) produced antinociception only up to day 45. After completing the 12-week training period, antinociception was no longer observed in rats submitted to the Ac protocol ( $\mathrm{Tr}-\mathrm{Ac})$. This could be explained by the fact that during a training program a constant release of endogenous opioid peptides occurs that may induce the development of tolerance (16). Another possible explanation is that the clearance of plasma $\beta$-endorphin following exercise may be increased by higher cardiac output and/or by adaptation to stress induced by training.

Although certain pain management programs prescribe aerobic exercises, it is important to consider that a combination of both (aerobic and resistance exercises) or even single sessions of resistance exercise alone can provide pain relief and are useful for treating patients unable to engage in high intensity and long duration aerobic exercise programs.

\section{Acknowledgments}

Research supported by CNPq and CAPES.

\section{References}

1. Jackson JK, Shepherd TR, Kell RT. The influence of periodized resistance training on recreationally active males with chronic nonspecific low back pain. J Strength Cond Res 2010 (in press).

2. Ritti-Dias RM, Wolosker N, de Moraes Forjaz CL, Carvalho CR, Cucato GG, Leao PP, et al. Strength training increases walking tolerance in intermittent claudication patients: randomized trial. J Vasc Surg 2010; 51: 89-95.

3. Camargo PR, Haik MN, Ludewig PM, Filho RB, MattielloRosa SM, Salvini TF. Effects of strengthening and stretching exercises applied during working hours on pain and physical impairment in workers with subacromial impingement syndrome. Physiother Theory Pract 2009; 25: 463-475.
4. Galdino GS, Cortes SF, Duarte ID, Perez AC. Involvement of the nitric oxide/(C)GMP/K(ATP) pathway in antinociception induced by exercise in rats. Life Sci 2010; 86: 505-509.

5. Bement MK, Sluka KA. Low-intensity exercise reverses chronic muscle pain in the rat in a naloxone-dependent manner. Arch Phys Med Rehabil 2005; 86: 1736-1740.

6. Tamaki T, Uchiyama S, Nakano S. A weight-lifting exercise model for inducing hypertrophy in the hindlimb muscles of rats. Med Sci Sports Exerc 1992; 24: 881-886.

7. Delorme TL, Watkins AL. Technics of progressive resistance exercise. Arch Phys Med Rehabil 1948; 29: 263-273.

8. Randall LO, Selitto JJ. A method for measurement of analgesic activity on inflamed tissue. Arch Int Pharmacodyn Ther 
1957; 111: 409-419.

9. Bartholomew JB, Lewis BP, Linder DE, Cook DB. Postexercise analgesia: replication and extension. J Sports Sci 1996; 14: 329-334.

10. Koltyn KF, Arbogast RW. Perception of pain after resistance exercise. Br J Sports Med 1998; 32: 20-24.

11. Anshel $M H$, Russell KG. Effect of aerobic and strength training on pain tolerance, pain appraisal and mood of unfit males as a function of pain location. J Sports Sci 1994; 12: 535-547.

12. Koltyn KF. Analgesia following exercise: a review. Sports Med 2000; 29: 85-98

13. Schmitt TK, Mousa SA, Brack A, Schmidt DK, Rittner HL, Welte $\mathrm{M}$, et al. Modulation of peripheral endogenous opioid analgesia by central afferent blockade. Anesthesiology 2003; 98: 195-202.

14. Pierce EF, Eastman NW, Tripathi HT, Olson KG, Dewey WL. Plasma beta-endorphin immunoreactivity: response to resistance exercise. J Sports Sci 1993; 11: 499-502.

15. Kraemer WJ, Dziados JE, Marchitelli LJ, Gordon SE, Harman EA, Mello R, et al. Effects of different heavy-resistance exercise protocols on plasma beta-endorphin concentrations. J Appl Physiol 1993; 74: 450-459.

16. Mathes WF, Kanarek RB. Wheel running attenuates the antinociceptive properties of morphine and its metabolite, morphine-6-glucuronide, in rats. Physiol Behav 2001; 74: 245-251. 